\title{
NÍVEL DE (IN)SATISFAÇÃO CORPORAL DE MULHERES PRATICANTES DE TREINAMENTO EM CIRCUITO
}

\author{
Gabriella Nelli Monteiro \\ Universidade Federal de Mato Grosso, Cuiabá, Mato Grosso, Brasil \\ Schelyne Ribas da Silva \\ Universidade Federal de Mato Grosso, Cuiabá, Mato Grosso, Brasil \\ Tatiane Mazzardo \\ Universidade Federal de Mato Grosso, Cuiabá, Mato Grosso, Brasil \\ Nayanne Dias Araújo \\ Universidade Federal de Mato Grosso, Cuiabá, Mato Grosso, Brasil \\ Layla Maria Campos Aburachid \\ Universidade Federal de Mato Grosso, Cuiabá, Mato Grosso, Brasil
}

\begin{abstract}
Resumo
O objetivo do estudo foi identificar o nível de (in)satisfação corporal e o percentual de gordura de mulheres praticantes do treinamento em circuito. A amostra constituiu-se de 110 mulheres com faixa etária entre 12 e 60 anos. Utilizou-se a silhuetas Stunkard para a identificação das silhuetas atual e desejada e a bioimpedância On-Rom 2006 para análise do percentual de gordura. Verificou-se que as mulheres desejam silhuetas menos volumosas e que, em todos os agrupamentos por idade, a maioria das mulheres se encontrava em sobrepeso e insatisfeita com sua imagem corporal. Verificou-se também associação entre o percentual de gordura e a (in)satisfação corporal $(\mathrm{p}=0,025)$ e que mesmo as mulheres consideradas eutróficas desejam silhuetas menores que a atual.
\end{abstract}

Palavras-chave: Imagem corporal. Perda de peso. Atividade física.

\section{Introdução}

A imagem corporal pode ser determinada como uma organização multidimensional que relata extensivamente as representações internas da estrutura corporal e da aparência física, em relação a nós mesmos e aos outros (DAMASCENO et al., 2006). Segundo Smolack e Levine (2001), a imagem corporal se compõe de dois componentes: a estima e a insatisfação corporal.

A primeira refere-se ao quanto um indivíduo gosta ou não de seu corpo de forma global, a qual pode incluir outros aspectos além do peso e da forma do corpo, como cabelos, rosto, entre outros. A segunda é um componente da dimensão atitudinal e refere-se à avaliação subjetiva negativa do próprio corpo (CASH; SMOLAK, 2011; FORTES et al., 2014)

Neste sentido, a utilização do termo imagem corporal é uma maneira de padronizar os diferentes componentes que a integram, dentre eles, a satisfação com o peso, a acurácia com a percepção da estatura, satisfação corporal, avaliação da aparência, orientação da aparência, 
estima corporal, corpo ideal, padrão de corpo, esquema corporal, percepção corporal, distorção corporal e desordem da imagem corporal, entre outros (DAMASCENO et al., 2006).

Notoriamente, estudos demonstram que este construto pode interferir até mesmo no convívio com a sociedade, principalmente no quesito aceitação. Tal fato pode ser observado com maior frequência entre os adolescentes, podendo influenciar no desenvolvimento desses indivíduos e até causar algum tipo de psicopatologia, estendendo-se à idade adulta (NICHOLLS; VINER, 2005; GOUVEIA et al, 2014; NEVES et al., 2017).

Fabrin et al. (2013) afirmam que a insatisfação corporal pode estar relacionada aos aspectos psicológicos, culturais, afetivos e ambientais que limitam o indivíduo a ter experiências corporais otimistas. Também está ligada à baixa autoestima e a limitações no desempenho psicossocial, associando-se a quadros depressivos e podendo também estar associada a transtornos alimentares (TRICHES; GIUGLIANE, 2007). Quando um indivíduo, mesmo estando com um peso adequado ou abaixo do peso ideal, costuma se sentir gordo e desproporcional é que se denomina de distorção de imagem corporal (BRANCO; HILÁRIO; CINTRA, 2006).

Geralmente, os indivíduos superestimam ou subestimam as formas ou o tamanho de seus corpos. Vieira (2011) aponta que as meninas adolescentes apresentaram uma tendência a subestimar e os meninos a superestimar seu próprio peso, colocando em destaque as meninas que apresentaram maior índice de insatisfação. Isso pode estar associado à pressão midiática ou a influências sociais que cultivam a cultura da busca de um corpo ideal, o que ocorre principalmente entre as mulheres (DAMASCENO et al., 2006).

A busca incessante por uma melhor aparência física é um fenômeno sociocultural muitas vezes mais significativo que a própria satisfação econômica, afetiva ou profissional (CATTARIN et al., 2000).

Neste sentido a insatisfação com o próprio corpo muitas vezes leva as pessoas a praticarem exercícios físicos, cada qual com seu objetivo particular, seja para entrar em forma, seja para melhorar sua qualidade de vida ou simplesmente melhorar sua disposição diária (FERMINO; PEZZINI; REIS, 2010).

Por ser um tipo de prática em voga no âmbito das academias, o treinamento em circuito, segundo Guilherme e Júnior (2006), pode ser uma boa ferramenta utilizada como meio para reduzir o peso corporal de indivíduos insatisfeitos com a própria imagem, pois este pode desenvolver diversas capacidades físicas relacionadas tanto a saúde como ao desempenho. Os treinos podem ser dinâmicos e envolver várias modalidades dentro do circuito, estando de acordo com as necessidades e os gostos dos alunos (TUBINO; MOREIRA, 2003).

Nesta perspectiva, o objetivo do estudo foi identificar o nível de (in)satisfação corporal, a imagem corporal e o percentual de gordura $(\% \mathrm{G})$ de mulheres praticantes do treinamento em circuito e a associação entre o \%G e (in)satisfação corporal e imagem corporal.

\section{Materiais e métodos}

A amostra foi composta de 110 mulheres $(36 \pm 13,69$ anos) matriculadas em uma academia para a prática de treinamento em circuito, com frequência de três aulas por semana e 40 minutos de duração por treino. Os grupos etários foram previamente estabelecidos, com base no estudo de Tessmer et al. (2006). Sendo assim, as faixas etárias foram agrupadas por: 12 a 19 anos; 20 a 29 anos; 30 a 39 anos; 40 a 49 anos; 50 a 59 anos e 60 anos acima.

O cálculo amostral realizado por descrição da população levou em conta a proporção de $70 \%$ da população, coeficiente de confiança de $95 \%$ e intervalo tolerável de $5 \%$. O tamanho da amostra ajustado foi aplicado, já que a população da academia onde os dados foram coletados era conhecida pelos pesquisadores. No estabelecimento quantitativo dos grupos por faixa etária, o teste do sinal foi aplicado para prevenir diferenças estatisticamente significati- 
vas entre as proporções. O projeto foi aprovado pelo Comitê de Ética em Pesquisa (314/2010) da Universidade Estadual do Centro-Oeste - UNICENTRO.

Para a avaliação do nível de (in)satisfação corporal utilizou-se como instrumento a Escala de Silhuetas de Stunkard et al. (1983), a qual representa um continuum desde a magreza (silhueta 1) até a obesidade (silhueta 9). Nesta escala, o indivíduo elege o número da silhueta que considera semelhante à sua aparência corporal atual e o número da silhueta que acredita ser mais condizente à sua aparência corporal ideal. O grau de satisfação com a silhueta corporal é obtido pela subtração entre a silhueta corporal ideal e a silhueta corporal atual. Se esta variação for igual a zero, os indivíduos são classificados como satisfeitos e, se diferente de zero, como insatisfeitos. Uma diferença positiva é considerada insatisfação por excesso de peso (desejo de diminuir o tamanho da silhueta) e uma diferença negativa representa a insatisfação pela magreza (desejo de aumentar a silhueta).

Para a avaliação do percentual de gordura, foi utilizado um aparelho de bioimpedância On-Rom2006. Para a avaliação, as mulheres foram orientadas a não ingerir líquidos nas duas horas antecedentes ao exame. Também não poderiam estar em período menstrual nem ter praticado atividades físicas vigorosas no dia do exame e teriam de ficar em repouso por cinco minutos antes do exame. No ato da avaliação, ficaram descalças e seguraram o aparelho com as mãos, mantendo os joelhos e cotovelos semiflexionados.

Os dados foram analisados por meio de frequência absoluta e relativa e do teste estatístico qui-quadrado e exacto de fisher para apontar a associação entre as variáveis. O programa Excel para Windows foi utilizado para a tabulação dos dados e estes foram tratados pelo programa Statistical Package for the Social Science (SPSS), versão 18, com valor de significância estabelecido em $\mathrm{p} \leq 0,05$.

\section{Resultados}

O primeiro resultado diz respeito à imagem corporal obtida por meio da escolha de silhuetas. Na Figura 1, observou-se que as mulheres desejam diminuir sua silhueta corporal, pois a silhueta atual eleita com maior frequência foi a de número 4 , seguida pela de número 5 . A figura mais desejada foi a de número 3, seguida pela figura de número 2.

Figura 1. Perfil geral das silhuetas atuais e desejadas.

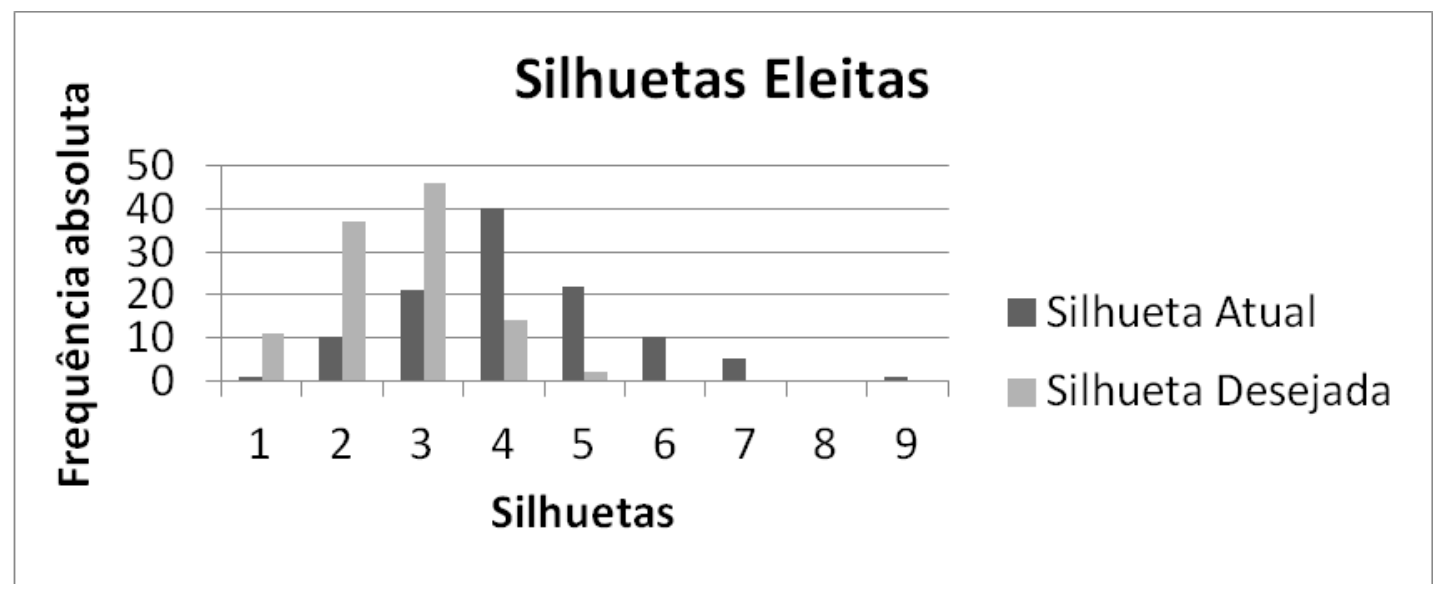

Fonte: Elaborado pelos autores, 2017

Os resultados do perfil da amostra (Tabela 1 a seguir) demonstraram altos percentuais para as classificações do \% G sobrepeso/obeso, seguido de valores acima do padrão de $20 \%$ por amostra, referenciados pela Organização Mundial de Saúde (OMS). Ainda em relação à 
(in)satisfação corporal, evidenciou-se que a grande maioria das mulheres do estudo $(93,6 \%)$ está insatisfeita com o seu volume corporal, sendo que $86,4 \%$ desejam perder peso, alcançando uma silhueta menor do que a atual. Em contrapartida, 7,3\% das mulheres do estudo desejam obter uma silhueta maior do que a atual.

Tabela 1. Resultados gerais do perfil da amostra.

\begin{tabular}{llll}
\hline & Classificação & $F$ & $\%$ \\
\hline$\% \mathrm{G}$ & Eutrófico & 8 & 7,3 \\
& Sobrepeso & 102 & 92,7 \\
(in)satisfação & Satisfeito & 7 & 6,4 \\
& Ganhar peso & 8 & 7,3 \\
Imagem Corporal & Perder peso & 95 & 86,4 \\
& Satisfeito & 7 & 6,4 \\
& Insatisfeito & 103 & 93,6 \\
\hline
\end{tabular}

Fonte: Elaborado pelos autores, 2017

Evidencia-se na Tabela 2 que, na faixa etária a partir dos 20 anos, os resultados de \% $\mathrm{G}$ aumentam sobremaneira e se classificam, em sua maioria, acima do normal. Todas as mulheres acima da faixa etária dos 50 anos encontram-se com o \%G considerado "sobrepeso". Nos dados do agrupamento da faixa etária mais jovem (12 a 19 anos), 100\% das mulheres se encontraram insatisfeitas, entretanto $40 \%$ delas estavam dentro dos padrões normais de saúde (eutrófico).

Tabela 2. Frequência do percentual de gordura em relação aos grupos etários

\begin{tabular}{lcc}
\hline Faixa etária & \multicolumn{2}{c}{$\%$ Gordura } \\
\hline & Eutrófico & Sobrepeso \\
\hline $12-19$ anos & $04(40 \%)$ & $f(\%)$ \\
$20-29$ anos & $03(10 \%)$ & $27(90 \%)$ \\
$30-39$ anos & $00(0 \%)$ & $28(100 \%)$ \\
$40-49$ anos & $01(4,3 \%)$ & $22(95,7 \%)$ \\
$50-59$ anos & $00(0 \%)$ & $14(100 \%)$ \\
60 acima & $00(0 \%)$ & $05(100 \%)$ \\
\hline
\end{tabular}

Fonte: Elaborado pelos autores, 2017

Na Tabela 3, se apresentam as frequências absolutas e relativas referentes à imagem corporal e à (in)satisfação relacionada às faixas etárias.

Tabela 3. Frequência da imagem corporal e das subescalas da (in)satisfação em relação aos grupos etários

\begin{tabular}{llllll}
\hline Faixa etária & \multicolumn{2}{l}{ (In)satisfação } & \multicolumn{3}{c}{ Imagem corporal } \\
\hline & Satisfeito & Ganhar peso & Perder peso & Insatisfeito & Satisfeito \\
\hline$f(\%)$ & $f(\%)$ & $f(\%)$ & $f(\%)$ & $f(\%)$ \\
\hline
\end{tabular}




\begin{tabular}{llllll}
\hline $12-19$ anos & $0(0 \%)$ & $0(0 \%)$ & $10(100 \%)$ & $10(100 \%)$ & $0(0 \%)$ \\
$20-29$ anos & $1(3,3 \%)$ & $3(10,0 \%)$ & $26(86,7 \%)$ & $29(96,7 \%)$ & $1(3,3 \%)$ \\
$30-39$ anos & $1(3,6 \%)$ & $1(3,6 \%)$ & $26(92,9 \%)$ & $27(96,4 \%)$ & $1(3,6 \%)$ \\
$40-49$ anos & $2(8,7 \%)$ & $4(17,4 \%)$ & $17(73,9 \%)$ & $21(91,3 \%)$ & $2(8,7 \%)$ \\
$50-59$ anos & $1(7,1 \%)$ & $0(0 \%)$ & $13(92,9 \%)$ & $13(92,9 \%)$ & $1(7,1 \%)$ \\
60 acima & $2(40 \%)$ & $0(0 \%)$ & $3(60 \%)$ & $3(60 \%)$ & $2(40 \%)$ \\
\hline
\end{tabular}

Fonte: Elaborado pelos autores, 2017

Verificou-se, na Tabela 3, que a somatória das mulheres satisfeitas com sua silhueta atual é de $62,7 \%$ e $86,4 \%$ apresentam-se insatisfeitas com sua silhueta atual e gostariam que fosse menor que a atual (perder peso), representando 95 mulheres investigadas. Dentre as mulheres da faixa etária entre 20 e 49 anos, $31 \%$ delas desejaram aumentar sua silhueta corporal (ganhar peso).

No que concerne às análises de associação entre as variáveis \% G com in(satisfação) corporal e imagem corporal, a Tabela 4 apresenta associação significativa entre o \% G e a (in)satisfação corporal para todas as mulheres da amostra $(\mathrm{p}=0,025)$. $\mathrm{O}$ fato de se enquadrar em um \% G "sobrepeso" se associa à insatisfação para a perda do peso. Entre as duas variáveis analisadas, as mulheres classificadas como eutróficas também apresentaram (in)satisfação corporal, o que refletiu para mulheres sobrepesadas. Porém, sete mulheres sobrepesadas $(6,9 \%)$ demonstraram satisfação com sua silhueta corporal. Verifica-se também que $88,2 \%$ de toda a amostra classificou-se como insatisfeita e desejava perder peso.

Tabela 4. Associação entre percentual de gordura e as subescalas da classificação da imagem corporal.

\begin{tabular}{|c|c|c|c|c|}
\hline & In(satisfac & & & \\
\hline$\%$ Gordura & Satisfeita & Ganhar peso & Perder peso & $\mathrm{p}$ \\
\hline$f(\%)$ & $f(\%)$ & $f(\%)$ & $f(\%)$ & \\
\hline Eutrófico & $0(0 \%)$ & $3(37,5 \%)$ & $5(62,5 \%)$ & \multirow{2}{*}{$0,025^{*}$} \\
\hline Sobrepeso & $7(6,9 \%)$ & $5(4,9 \%)$ & $90(88,2 \%)$ & \\
\hline
\end{tabular}

exacto de fisher $(* \mathrm{p} \leq 0,05)$

Fonte: Elaborado pelos autores, 2017

\section{Discussão}

A busca por uma melhor aparência física por praticantes de atividade física é um fenômeno sociocultural às vezes mais significativo do que a própria satisfação econômica, afetiva ou profissional (JUNIOR; JUNIOR; SILVEIRA, 2013). Os principais motivos pelos quais as pessoas aderem a programas de atividade física são a insatisfação com o próprio corpo ou mesmo com a imagem que se tem dele e a valorização exacerbada de baixos níveis de gordura corporal (DAMASCENO et al, 2006).

Segundo Korn et al. (2013), as mulheres são mais insatisfeitas que os homens no que se refere à percepção da autoimagem corporal e à prática de exercícios físicos. Elas perseguem o ideal de magreza, ao contrário dos homens, que buscam ser mais fortes e volumosos (CLAUMANN et al., 2014).

Na reflexão feita por Gonçalves, Campana e Tavares (2012), programas de atividade física bem elaborados podem exercer influência na imagem corporal tanto de homens como mulheres para alcançar os corpos que idealizam, levando-se em consideração a individualidade do sujeito. Entretanto, a prática de atividade física, em alguns casos, pode resultar em con- 
sequências negativas, aumentando, nas mulheres, a preocupação com a magreza (KAKESHITA; ALMEIDA, 2006).

Não obstante, os resultados observados apontam que a amostra, independentemente da faixa etária, encontra-se, com maior frequência, na classificação sobrepesada e insatisfeita com a sua forma física. Este sentimento de (in)satisfação corporal vai ao encontro dos estudos de Saur e Pasian (2008) e Tessmer et al. (2006), que afirmam que o percentual de insatisfação corporal por amostra frequentemente varia entre $60 \%$ e $100 \%$.

Mais especificamente, em faixas etárias mais altas, o presente estudo corrobora os resultados do estudo de Ferreira et al. (2014), que avaliou mulheres com média de 71 anos de idade participantes de um curso universitário e diagnosticou, por meio do mesmo instrumento utilizado nesta pesquisa, que a maioria das mulheres avaliadas $(74 \%)$ apresentava insatisfação com sua aparência corporal.

Esta pesquisa ainda ratifica os estudos de Souto et al. (2016), que compararam a imagem corporal de 300 mulheres, entre 20 e 83 anos, divididas em adultas, meia-idade e idosas praticantes e não praticantes de hidroginástica. Observou-se uma diferença significativa entre imagem corporal atual e desejada em todos os grupos $(\mathrm{p}=0.001)$, ou seja, insatisfação com a imagem corporal.

Tendo como referência estudos que verificaram o nível de (in)satisfação e prática de atividade física, Damasceno et al. (2005) quantificaram o nível de insatisfação com a imagem corporal de praticantes de caminhada. Os resultados apontaram que as mulheres estão insatisfeitas com a sua silhueta atual e desejam silhuetas menos volumosas. Estes dados corroboram ainda os achados desta pesquisa e os relatos do estudo de Oliveira et al. (2017).

A satisfação com o próprio corpo parece estar relacionada às exigências sociais de aparência e magreza; as pessoas parecem tender, de forma estereotipada, a avaliar a imagem corporal em função de normas culturais de peso ideal (FERMINO; PEZZINI; REIS, 2010). Resultados semelhantes foram identificados nesta pesquisa, pois $40 \%$ das jovens pertencentes à faixa etária entre 12 e 19 anos, consideradas com \% G normal, também desejam uma silhueta menos volumosa, ou seja, elas subestimam sua forma física, assim como mostram os estudos de Branco, Hilário e Cintra (2006) e Alves et al. (2017).

Em contrapartida, os apontamentos da Figura 1 balizaram que poucas mulheres foram alocadas entre as silhuetas de número 5 e 9, que representam indivíduos com obesidade. A Tabela 1 apresentou uma predominância de mulheres sobrepesadas e com classificação obesa no índice de massa corporal. Esse fato demonstra uma subestimação das silhuetas atuais por parte das mulheres, reforçando as afirmações dos estudos de Kakeshita e Almeida (2006), Rech, Araújo e Vanat (2010) e Alves et al. (2017), com estudantes universitárias que, em sua maioria, foram classificadas como obesas, mas que independentemente do índice de massa corporal tenderam a subestimar o tamanho corporal. Os resultados encontrados no presente estudo também corroboram Pinheiro e Giugliani (2006), Tessmer et al. (2006), Alvarenga et al. (2010) e Vieira (2011), que, além de evidenciar que a maioria das escolares e universitárias gostaria de se apontar menores do que sua figura atual, elas, mesmo classificadas como eutróficas, também apontaram figuras menores em volume.

Evidências apontam que, em adolescentes, a insatisfação corporal está diretamente associada ao desenvolvimento de problemas e distúrbios alimentares segundo Triches e Giugliane (2007), Alves et al. (2008) e Kunzler et al. (2014), sendo que os principais fatores de risco associados à preocupação com a aparência corporal têm sido a exposição à mídia, que impõe cada vez mais padrões de beleza caracterizados pela magreza excessiva, e a pressão da família e dos amigos (BERG et al., 2007).

O estudo qualitativo de Oliveira, Santos e Rocha (2016) teve como objetivo identificar como adolescentes escolares do sexo feminino de escolas públicas relacionam o aspecto físico com as relações sociais e a imagem corporal. Participaram 48 adolescentes com média de 
$15,5(+1,2)$ anos de idade, separadas em oito grupos que seguiram um roteiro semiestruturado de dez questões. Após a análise das entrevistas, houve alta rejeição ao corpo, identificando a utilização de dietas com ênfase na restrição alimentar e na utilização de cirurgia plástica para alcançar o corpo ideal. Nesta perspectiva, os resultados do presente estudo corroboram os apresentados pelos autores supracitados quando se referem à rejeição ou à insatisfação corporal das mulheres em relação a suas silhuetas, levando-se em conta a idade.

Essas evidências auxiliam nas discussões das diferenças nos níveis de insatisfação corporal em relação à idade. Sendo assim, é plausível inferir que uma maior carga de exigência estética possa incidir sobre mulheres mais velhas, justificando a escolha de silhuetas mais magras (DITTMAR; HALLIWELL; IVE, 2006).

\title{
Conclusão
}

Considerando o objetivo do presente estudo, verificou-se uma alta frequência de insatisfação corporal e sobrepeso entre as mulheres, independentemente da faixa etária avaliada.

Diante disso, observa-se a relevância do estudo, tendo em vista que assuntos sobre transtornos ligados ao peso e à alimentação ainda permeiam com frequência os estudos científicos na atualidade. Torna-se de suma importância que os profissionais da área da saúde tenham clareza sobre esse assunto, já que trabalham diariamente com técnicas corporais de culto ao corpo, desenvolvendo papéis na formação de valores educacionais, socioculturais e até mesmo psicossociais.

Sugere-se a realização de novos estudos utilizando como amostra mulheres de diferentes faixas etárias e modalidades praticadas em academias, bem como a utilização de questionários que avaliem a (in)satisfação corporal de adultos, a fim de enriquecer as discussões acerca desse assunto.

\section{BODY (IN) SATISFACTION LEVEL IN WOMEN WHO PRACTICE TRAINING CIRCUIT}

\begin{abstract}
The study goal was to identify the level of Body (in)Satisfaction and fat percentage of women who practice training circuit. The sample consisted of 110 women aged 12-60 years. It was used measuring instrument for silhouettes Stunkard for identification of current and desired silhouettes and bioimpedance On- Rom 2006 for analysis of the percentage of fat. It was found that women wish silhouettes less bulky and that in all age-groups most women were in overweight and dissatisfied with their body image. It was also found significance between the percentage of fat and (in) body satisfaction $(\mathrm{p}=0,025)$ and that even women considered normal weight wish silhouettes smaller than the current.
\end{abstract}

Keywords: Body Image. Weight loss. Physical activity.

\section{NIVEL DE (IN)SATISFACCIÓN DEL CUERPO DE LAS MUJERES QUE ENTRE- NAN EN CIRCUITO}

\author{
Resumen \\ El objetivo del estudio fue identificar el nivel de (in)satisfacción corporal y el porcentaje de \\ grasa de mujeres que se entrenan en circuito. La muestra está constituida por 110 mujeres con \\ edades comprendidas entre los 12 y los 60 años. Se utilizaron las siluetas Stunkard para iden- \\ tificar las siluetas reales y las deseadas y la bioimpedancia On- Rom 2006 para el análisis del
}


porcentaje de grasa. Se encontró que las mujeres desean siluetas menos voluminosas y que en todos los grupos de edad la mayoría de las mujeres estaba con sobrepeso e infeliz con su imagen corporal. Se observó también la asociación entre el porcentaje de grasa y la (in)satisfacción corporal $(\mathrm{p}=0,025)$ y que incluso las mujeres consideradas de peso normal quieren siluetas menores que las actuales.

Palabras clave: Imagen corporal. Pérdida de peso. Actividad física.

\section{Referências}

ALVARENGA M. S.; PHILIPPI S.T.; LOURENÇO B. H.; SATO P. M.; SCAGLIUSI F.B. Insatisfação com a imagem corporal em universitárias brasileiras. Jornal Brasileiro de Psiquiatria, n. 59, v.1, 2010. Disponível em:

<http://www.scielo.br/scielo.php?script=sci_arttext\&pid=S0047-20852010000100007>. Acesso em: 10 maio 2016.

ALVES, E.; VASCONCELOS, F. A. G.; CALVO, M. C. M.; NEVES, J. Prevalência de sintomas de anorexia nervosa e insatisfação com a imagem corporal em adolescentes do sexo feminino do município de Florianópolis, Santa Catarina, Brasil. Cadernos de Saúde Pública, Rio de Janeiro, n. 24, p. 503-512, 2008. Disponível em:

$<$ http://www.scielo.br/scielo.php?script=sci_arttext\&pid=S0102-311X2008000300004>.

Acesso em: 15 maio 2016.

ALVES, F. R.; SOUZA, E. A.; PAIVA, C. S.; TEIXEIRA, F. A. A. Insatisfação com a imagem corporal e fatores associados em universitários. Cinergis, Santa Cruz do Sul, v. 18, n. 3, p. 210-215, 2017. Disponivel em:

$<$ https://online.unisc.br/seer/index.php/cinergis/article/view/9037/6100>. Acesso em: 24 out. 2017.

BERG, P. V. D.; PAXTON, S. J.; KEERY, H.; PAREDE, M.; GUO, J.; NEUMARKSZTAINER, D. Body dissatisfaction and body comparison with media images in males and females. Body Image, Norfolk, v. 4, n. 3, p. 257-268, 2007. Disponível em: <http://www.sciencedirect.com/science/article/pii/S1740144507000435>. Acesso em: 9 maio 2016.

BRANCO, L. M.; HILÁRIO, M. O. E.; CINTRA, I. P. Percepção e satisfação corporal em adolescentes e a relação com seu estado nutricional. Revista de Psiquiatria Clínica, v. 33, n. 6, 2006. Disponível em: < http://www.repositorio.unifesp.br/handle/11600/2847>. Acesso em: 15 de maio 2016.

CASH, T.F; SMOLAK, L. Body image: A handbook of science, practice, and prevention. 2 ed. New York: The Guilford Press, 2011.

CATTARIN, J.; WILLIAMS, R.; THOMAS, C. M.; THOMPSON, K. J. Body image, mood, and televised images of attractiveness: the role of social comparison. The British Journal of Medical Psychology, v. 19, n. 2, p. 220-239, 2000. Disponível em: <http://guilfordjournals.com/doi/abs/10.1521/jscp.2000.19.2.220>. Acesso em: 13 nov. 2017.

ClAUMANN, G. S.; PEREIRA, E. F.; INÁCIO, S.; SANTOS, M. C.; MARTINS, A.C.; PELEGRINI, A. Satisfação com a imagem corporal em acadêmicos ingressantes em cursos de 
educação física. Revista da Educação Física/UEM, v. 25, n. 4, p. 575-583, 2014. Disponível em:

<http://www.periodicos.uem.br/ojs/index.php/RevEducFis/article/view/23456 >. Acesso em: 24 out. 2017.

DAMASCENO, V. O.; LIMA, J. R. P.; VIANNA, J. M.,;VIANNA, V. R. A.; NOVAES, J.S. Tipo físico ideal e satisfação com a imagem corporal de praticantes de caminhada. Revista Brasileira de Medicina do Esporte, v. 11, n. 3, 2005. Disponível em: <http://www.scielo.br/scielo.php?script=sci_arttext\&pid=S1517-86922005000300006>. Acesso em: 11 maio 2016.

DAMASCENO, V. O.; VIANNA, V. R. A.; VIANNA, J. M.; LACIO, M., LIMA; J. R. P.; NOVAES, J. S. Imagem corporal e corpo ideal. Revista Brasileira. Ciência e Movimento, v. 14, n. 2, p. 87-94, 2006. Disponível em:

<https://www.researchgate.net/publication/236019947_Imagem_corporal_e_corpo_ideal>. Acesso em: 13 maio 2016.

DITTMAR, H.; HALLIWELL, E.; IVE, S. Does Barbie make girls want to be thin? The effect of experimental exposure to images of dolls on the body image of 5 to 8 year old girls. Dev. Psychol, v. 42, n. 2, p. 283-92, 2006. Disponível em:

<http://psycnet.apa.org/journals/dev/42/6/1258/>. Acesso em: 10 maio 2016.

FABRIN, T. K.; FIN, G.; BARETTA, M.; BARETTA, E. Percepção da imagem corporal e percentual de gordura em adolescente do gênero feminino. Unoesco \& Ciencia - ACBS, Joaçaba, v. 4, n. 2, p. 195-202, 2013. Disponível em:

$<$ https://editora.unoesc.edu.br/index.php/acbs/article/viewFile/3615/pdf_17>. Acesso em: 13 de nov. 2017.

FERMINO, R. C.; PEZZINO, M. R.; REIS, R. S. Motivos para a prática de atividade física e imagem corporal em frequentadores de academia. Revista Brasileira de Medicina e Esporte, v. 16, n.1, p. 18-23, 2010. Disponível em: <http://www.scielo.br/pdf/rbme/v16n1/a03v16n1.pdf>. Acesso em: 23 out. 2017.

FERREIRA, A. A.; MENEZES, M. F. G.; TAVARES, E. L.; NUMES, N. C.; SOUZA, F. P.; ALBUQUERQUE, N. A. F.; PINHEIRO, M. A. M. Estado nutricional e autopercepção da imagem corporal de idosas de uma Universidade Aberta da Terceira Idade. Revista Brasileira de Geriatria e Gerontologia, v. 17, n. 2, p. 289-301, 2014. Disponível em:

< http://www.scielo.br/pdf/rbgg/v17n2/1809-9823-rbgg-17-02-00289.pdf>. Acesso em: 24 out. 2017.

FORTES, L. S., CIPRIANI, F. M.; COELHO, F. D.; PAES, S. T.; FERREIRA, M. E. C. A autoestima afeta a insatisfação corporal em adolescentes do sexo feminino? Revista Paulista de Pediatria, v. 32, n. 3, p. 236-240, 2014. Disponível em: $<$ http://www.scielo.br/scielo.php?pid=S0103-

05822014000300236\&script=sci_abstract\&tlng=pt >. Acesso em: 13 nov. 2017.

GONÇALVES, C.O.; CAMPANA, A.N.; TAVARES, M.C. Influência da atividade física na imagem corporal: uma revisão bibliográfica. Motricidade, Vila Real, v. 8, n.2, p. 70-82, 2012 . Disponível em <http://www.scielo.mec.pt/scielo.php?script=sci_arttext\&pid=S1646107X2012000200008\&lng=pt\&nrm=iso>. Acesso em: 23 out. 2017. 
GOUVEIA, M. J.; FRONTINI, R.; CANAVARRO, M. C.; MOREIRA, H. Quality of life and psychological functioning in pediatric obesity: the role of body image dissatisfaction between girls and boys of different ages. Quality of Life Research, v. 9, n. 23, 2014. Disponível em: <https://link.springer.com/article/10.1007\%2Fs11136-014-0711-y>. Acesso em: 21 out. 2017.

GUILHERME, J. P. L. F.; JÚNIOR, T. P. S. Treinamento de força em circuito na perda e no controle corporal. Revista Conexão, v. 4, n. 2, p. 31-46, 2006. Disponível em: <http://www.luzimarteixeira.com.br/wp-content/uploads/2011/04/treinamento-em-circuito-eperda-de-peso.pdf $>$. Acesso em: 13 nov. 2017.

JUNIOR, M. P.; JUNIOR, W. C.; SILVEIRA, F.V. Percepção e distorção da auto imagem corporal em praticantes de exercício físico: a importância do exercício físico na imagem corporal. Revista Brasileira de Nutrição Esportiva. São Paulo, v. 42, n.7, p. 345-352, 2013. Disponível em: <http://www.rbne.com.br/index.php/rbne/article/view/410/394>. Acesso em: 23 out. 2017.

KAKESHITA, I. S.; ALMEIDA, S. S. Relação entre índice de massa corporal e a percepção da auto-imagem em universitários. Revista de Saúde Pública, v. 40, n. 3, p. 497-504, 2006. Disponível em: <http://www.revistas.usp.br/rsp/article/view/32066>. Acesso em: 14 maio 2016.

KORN, L.; GONEN, E.; SHAKED, Y.; GOLAN, M. Health perceptions, self and body image, physical activity and nutrition among undergraduate students in Israel. PLoS One, v. 8, n. 3, p. 1-7, 2013. Disponível em:

<http://journals.plos.org/plosone/article?id=10.1371/journal.pone.0058543>. Acesso em: 23 out. 2017.

KUNZLER, R. J.; MAMENTI, M.; BOFF, E. T. O.; BUSNELLO, M. B.; FRANZ, L. B. B. Percepção da imagem corporal entre estudantes adolescentes da educação básica. Salão do Conhecimento, v. 1, n. 2, 2014. Disponível em:

<https://www.publicacoeseventos.unijui.edu.br/index.php/salaoconhecimento/article/view/39 20/3295>. Acesso em: 24 out. 2017.

NEVES, C. M. et al. Imagem corporal na infância: uma revisão integrativa da literatura. Revista Paulista de Pediatria, São Paulo, v. 35, n. 3, p. 331-339, set. 2017. . Disponível em: <http://www.scielo.br/scielo.php?script=sci_arttext\&pid=S0103-05822017000300331 \&lng=en\&nrm=iso $>$. Acesso em: 19 nov. 2017.

NICHOLLS, D.; VINER, R. Eating disorders and weight problems. BMJ, v. 330, n. 7497, p. 950-953, 2005. Disponível em: <https://www.ncbi.nlm.nih.gov/pubmed/15845978>. Acesso em: 13 nov. 2017.

OLIVEIRA, A. R. C.; SANTOS, A. B.; ROCHA, L. F. Educação física escolar e imagem corporal em adolescentes: relatos de uma insatisfação. Investigação Qualidade em Saúde, v. 2, 2016. Disponível em: <http://proceedings.ciaiq.org/index.php/ciaiq2016/article/view/802〉. Acesso em: 25 jul. 2016. 
OLIVEIRA, G. L.; CONÇALVES, P. S. P.; OLIVEIRA, T. A. P.; FERNANDES, P. R.; FILHO, J. F. Avaliação da imagem corporal de mulheres praticantes de musculação, Revista Digital, Buenos Aires, v. 231, n. 22, 2017. Disponível em:

$<$ http://www.efdeportes.com/efd231/avaliacao-da-imagem-corporal-de-mulheresmusculacao.htm >. Acesso em: 24 out. 2017.

PINHEIRO, A. P.; GIUGLIANI, E. R. J. Body dissatisfaction in Brazilian schoolchildren: prevalence and associated factors. Revista de Saúde Pública, v. 40, n. 3, p. 489-496, 2006. Disponível em: http://www.scielo.br/scielo.php?script=sci_arttext\&pid=S003489102006000300018. Acesso em: 18 mai. 2016.

RECH, C. R.; ARAUJO, E. D. S.; VANAT, J. R. Autopercepção da imagem corporal em estudantes do curso de educação física. Revista Brasileira de Educação Física e Esporte, São Paulo, v. 24, n. 2, p. 285-292, $2010 . \quad$ Disponível em $<$ http://www.scielo.br/scielo.php?script=sci_arttext\&pid=S180755092010000200011\&lng=pt\&nrm=iso >. Acesso em: 23 out. 2017.

SAUR, A.M.; PASIAN, S.R. Satisfação com a imagem corporal em adultos de diferentes pesos corporais. Avaliação Psicológica, v. 7, n. 2, p. 199-209, 2008.

SMOLAK, L.; LEVINE, M. P. Imagem do corpo em crianças. In: THOMPSON, J. K., SMOLAK, L. (Ed.). Imagem do corpo, distúrbios alimentares e obesidade na juventude: avaliação, prevenção e tratamento. Washington (DC): American Psychological Association, 2001. p.41-66.

SOUTO, S. V. D.; NOVAES, J. DA S.; MONTEIRO, M. D.; NETO, G.L R.; CARVALHAL, M. I. M.; COELHO, E. Imagem corporal em mulheres adultas vs. meia idade e idosas praticantes e não praticantes de hidroginástica. Motricidade, v.12, n.1, p. 53-59, 2016. Disponível em: <http://revistas.rcaap.pt/motricidade/article/view/5000>. Acesso em: 14 maio 2016.

STUNKARD, A. J.; SORENSON, T.; SCHLUSINGER, F. Use of the danish adoption register for the study of obesity and thinness. In: KETY, S.S.; ROWLAND, L.P.; SIDMAN, R.L.; MAT-THYSSE, S.W. (Ed.). The genetics of neurological and psychiatric disorders. New York: Raven, 1983.

TESSMER, C. S.; SILVA, M. C.; PINHO, M. N.; GAZALLE, F. K.; FASSA, A.G. Insatisfação corporal em frequentadores de academia. Revista Brasileira de Ciência e Movimento, v. 14, n.1, p. 7-12, 2006. Disponível em:

$<$ https://portalrevistas.ucb.br/index.php/RBCM/article/viewFile/672/677>. Acesso em: 23 maio 2016.

TRICHES, R. M.; GIUGLIANE, E. R.J . Insatisfação corporal em escolares de dois municípios da região sul do Brasil. Revista de Nutrição, v. 20, n.2, 2007. Disponível em: http://www.scielo.br/scielo.php?script=sci_arttext\&pid=S1415-52732007000200001. Acesso em: 17 maio 2016.

TUBINO, M. J. G.; MOREIRA, S. B. Metodologia científica do treinamento desportivo. 13 ed. Rio de Janeiro: Shape, 2003. p. 312-316. 
VIEIRA, A. V. Relação do estado nutricional e satisfação com a imagem corporal entre escolares da rede pública de ensino de São Paulo. Revista Brasileira de Obesidade, Nutrição e Emagrecimento, São Paulo, v. 26, n. 5, p. 90-99, 2011. Disponível em:

< http://www.rbone.com.br/index.php/rbone/article/viewFile/222/217>. Acesso em: 24 out. 2017.

Recebido em: 01/08/2016

Revisado em: 06/11/2017

Aprovado em: 19/11/2017

Endereço para correspondência:

gnm cba@hotmail.com

Gabriella Nelli Monteiro

Universidade Federal do Mato Grosso

Av. Fernando Corrêa da Costa, 2367

Boa Esperança

78060-900 - Cuiabá, MT - Brasil 